\title{
On the Stationary Distribution of Speed and Location of Random Waypoint
}

\author{
Jean-Yves Le Boudec
}

October 12, 2004

\begin{abstract}
In "Stationary Distributions for the Random Waypoint Mobility Model" (TMC Vol 3 No 1), Navidi and Camp find the stationary distribution of the random waypoint model, with or without pause on a rectangular area. In this short note we show that, under the stationary regime, speed and location are independent.
\end{abstract}

Keywords. Mobility Model, Stationary Behaviour, Perfect Simulation, Random Waypoint

\section{Introduction}

In [3] Navidi and Camp find the stationary distribution of the random waypoint model, with or without pause on a rectangular area. In this short note we complement their result with the following additional finding.

In Sections 3 and 4 of [3], the authors find the stationary distributions of the speed and the location of the mobile, and use it to initialize a simulation in stationary regime. However, they do not derive the joint stationary distribution of speed and location. In fact, their simulation algorithm implicitly makes the assumption that speed and location sampled at an arbitrary time instant are independent, in the stationary regime; but it is entirely not obvious a priori whether this assumption is true or not. It is true that speed and next waypoint are independent when sampled at an arbitrary transition time, but this does not imply the same property at an arbitrary time instant. (A transition time is a time at which a waypoint is reached and a new trip is chosen). To be convinced, consider for example the location of path endpoints. When sampled at an arbitrary transition, the two points

*This material is presented to ensure timely dissemination of scholarly and technical work. Copyright and all right therein are retained by authors or by other copyright holders. All persons copying this information are expected to adhere to the terms and constraints invoked by each authors copyright. In most cases, these works may not be reposted without the explicit permission of the copyright holder. 
are independent, by construction. But when sampled at an arbitrary point in time, they are not (as witnessed by the joint density given in $\mathrm{Eq}(3)$ of [3]: it is more likely that the two endpoints are far apart).

We show, using formal derivations, that independence of speed and location at an arbitrary point in time does hold.

We obtain this result by using Palm calculus, a set of formulas that relate time averages to event averages, and can be used to explain more simply the original results of Navidi and Camp. For a quick overview of Palm calculus, see [2]; for a full fledged theory, see [1].

\section{Notation and Terms}

We use the same notation as Navidi and Camp but need some minor extensions, defined below.

$$
\begin{array}{ll}
M(t)=(X(t), Y(t)) & \begin{array}{l}
\text { Location of mobile at time } t \\
S(t)
\end{array} \\
\text { Numerical speed of mobile at time } t \\
\operatorname{Next}(t)=\left(X_{2}(t), Y_{2}(t)\right) & \text { Next Waypoint, counted from time } t \\
\operatorname{Prev}(t)=\left(X_{1}(t), Y_{1}(t)\right) & \text { Previous Waypoint, counted from time } t \\
T_{n} & \text { Time at which the } n \text {th waypoint is reached } \\
& \text { By convention, } T_{0} \leq 0<T_{1}<\ldots<T_{n}<\ldots \\
\left\|M_{1}-M_{0}\right\| & \text { Euclidian distance between the two points } M_{1} \text { and } M_{0}
\end{array}
$$

\section{Independence of Speed and Location in the Stationary Regime}

We first consider the random waypoint without pause (Section 3 in [3]). Assume the conditions for stationarity of the random waypoint model hold, namely, the average time spent between two waypoints is finite. Then:

Theorem 3.1 The numerical speed $S(t)$ and $(M(t), \operatorname{Prev}(t), N e x t(t))$ are independent under the time stationary distribution.

Proof. We use the inversion formula of Palm calculus [1, 2], which says that, if the process $Z(t)$ is jointly stationary with the random waypoint model:

$$
\mathbb{E}(Z(t))=\mathbb{E}(Z(0))=\lambda \mathbb{E}^{0}\left(\int_{0}^{T_{1}} Z(s) d s\right)
$$

In the equation, $\mathbb{E}^{0}$ is the "Palm expectation", which can be interpreted as the expectation, conditional to the event that the mobile has reached a waypoint at time $t=0 . T_{1}$ is the time at which the next waypoint will be reached, given that we are at a waypoint at time $0 . \lambda$ is the "intensity", i.e. the average number of transitions per time unit.

Now consider two bounded, arbitrary functions $\phi$ and $\psi$, where $\phi$ is a function of the numerical speed and $\psi$ of the triple $(M(t), \operatorname{Prev}(t), \operatorname{Next}(t))$. Apply the inversion formula and obtain:

$$
\mathbb{E}(\phi(S(t) \psi(M(t), \operatorname{Prev}(t), \operatorname{Next}(t)))
$$




$$
\begin{aligned}
& =\lambda \mathbb{E}^{0}\left(\int_{0}^{T_{1}} \phi\left(S_{0}\right) \psi\left(M_{0}+\frac{t}{T_{1}}\left(M_{1}-M_{0}\right), M_{0}, M_{1}\right) d t\right) \\
& =\lambda \mathbb{E}^{0}\left(\phi\left(S_{0}\right) \int_{0}^{T_{1}} \psi\left(M_{0}+\frac{t}{T_{1}}\left(M_{1}-M_{0}\right), M_{0}, M_{1}\right) d t\right)
\end{aligned}
$$

where $S_{0}$ is the constant speed in the interval $\left[T_{0}, T_{1}\right]$ and $M_{0}$ [resp. $M_{1}$ ] is the waypoint at time $t=T_{0}$ [resp. $T_{1}$ ]. By the change of variable in the integral $t \rightarrow t / T_{1}$ we obtain:

$$
\begin{aligned}
& =\lambda \mathbb{E}^{0}\left(\phi\left(S_{0}\right) T_{1} \int_{0}^{1} \psi\left(M_{0}+t\left(M_{1}-M_{0}\right), M_{0}, M_{1}\right) d t\right) \\
& =\lambda \mathbb{E}^{0}\left(\frac{\phi\left(S_{0}\right)}{S_{0}}\left\|M_{1}-M_{0}\right\| \int_{0}^{1} \psi\left(M_{0}+t\left(M_{1}-M_{0}\right), M_{0}, M_{1}\right) d t\right) \\
& =\lambda \mathbb{E}^{0}\left(\frac{\phi\left(S_{0}\right)}{S_{0}}\right) \mathbb{E}^{0}\left(\left\|M_{1}-M_{0}\right\| \int_{0}^{1} \psi\left(M_{0}+t\left(M_{1}-M_{0}\right), M_{0}, M_{1}\right) d t\right)
\end{aligned}
$$

The last equality is due to the independence of speed and waypoint under $\mathbb{E}^{0}$. Thus we have factored the joint expectation in two terms. By Lemma 4.1, shown in appendix, this proves independence.

The theorem justifies the sampling procedure in Section 3 of [3]: sample a speed and a location (with the auxiliary points $\operatorname{Prev}(t)$ and $\operatorname{Next}(t)$ ) independently and obtain a simulation in stationary regime at time 0 .

Second, we consider the model with pauses (Section 4 of [3]). Here, it can be shown, using the same inversion formula, that the distribution of simulation state given that the mobile is not paused is the same as the stationary distribution for the model without pause. The conditional independence of speed and location follows.

\section{Appendix}

Lemma 4.1 Two random variables $X$ and $Y$ are independent if for any two bounded functions $\phi$ and $\psi$ we can factor $\mathbb{E}(\phi(X) \psi(Y))=\mathcal{A}(\phi) \mathcal{B}(\psi)$ where $\mathcal{A}$ and $\mathcal{B}$ are deterministic mappings of the functions $\phi$ and $\psi$.

Proof. Assume the factorization holds. All we need to show is that

$$
\mathbb{E}(\phi(X) \psi(Y))=\mathbb{E}(\phi(X)) \mathbb{E}(\psi(X))
$$

Since the relation in the hypothesis is true for any $\psi$ we can replace $\psi$ by the constant function equal to 1 and obtain $\mathcal{A}(\phi) \mathcal{B}(1)=\mathbb{E}(\phi(X))$. Similarly $\mathcal{A}(1) \mathcal{B}(\psi)=\mathbb{E}(\psi(X))$. Replace both $\phi$ and $\psi$ by the constant 1 and obtain $\mathcal{A}(1) \mathcal{B}(1)=1$. By multiplying the first two relations we obtain $\mathcal{A}(\phi) \mathcal{B}(\psi)=\mathbb{E}(\phi(X)) \mathbb{E}(\psi(X))$, which shows Equation (1).

\section{Conclusion}

Using Palm calculus, it is easy to extend the results by Navidi and Camp to an arbitrary connected (not necessarily convex) area (instead of a rectangle). Further, it is also possible to do this without computing complicated geometric integrals such as the mean distance between two points: see [2]. 


\section{References}

[1] François Baccelli and Pierre Brémaud. Palm Probabilities and Stationary Queues. Springer LNS, 1987.

[2] Jean-Yves Le Boudec. Understand the simulation of mobility models with palm calculus. Technical Report EPFL/IC/2004/53, EPFL, 062004.

[3] William Navidi and Tracy Camp. Stationary distributions for the random waypoint model. IEEE Transactions on Mobile Computing, 3(1), 2004. 